\title{
Traducir las identidades africanas: una mirada mujerista
}

\section{Translating African Identities: a «womanist» Approach}

\author{
Isabel PASCUA FEBLES y Alba RODRíGUeZ-GARCía \\ Universidad de Las Palmas de Gran Canaria / Université Gaston Berger \\ isabel.pascua@ulpgc.es / alba-rodriguez.garcia@ugb.edu.sn
}

Recibido febrero-2019. Revisado: marzo-2019. Aceptado: mayo-2019.

\begin{abstract}
Resumen: El presente trabajo pretende realizar un análisis contrastivo de dos novelas de autoras poscoloniales africanas y sus respectivas traducciones. Estas novelas están escritas en lenguajes híbridos, africanos en su fondo y europeizados en su forma, a saber: el francés del Senegal de Ken Bugul y el inglés de Nigeria de Buchi Emecheta, lenguas coloniales de las que los autores africanos se han apropiado y han renovado, lenguas "still in full communion with its ancestral home but altered to suit new African surroundings» (Achebe 1965/1997, 348). Partimos de la premisa establecida en los estudios coloniales, según la cual los textos híbridos poscoloniales poseen particularidades lingüísticas muy específicas y numerosas referencias culturales (muchas de ellas en lenguas vernáculas como el wolof o el igbo) que suponen un escollo para su traducción. En nuestra hipótesis, no puede aplicarse una única tendencia o estrategia; se deben explorar posibilidades más allá de las teorías clásicas y de la dicotomía de Schleiermacher (1813/1992) o Venuti (1995/2004), con el fin de buscar un equilibrio entre las diferentes posibilidades traslativas. Desde una mirada mujerista, ilustraremos fragmentos de estas obras con el fin de analizar cómo la cultura, el lenguaje híbrido y la identidad africana son expuestas desde un prisma femenino, y estudiaremos el tratamiento traductológico que ciertos fragmentos ilustrativos de estas escrituras femeninas africanas han experimentado hacia el español.
\end{abstract}


Palabras clave: Traducción; hibridismo; literatura poscolonial africana; mujerismo; culturemas; identidad.

\begin{abstract}
This article presents a contrastive analysis of two novels by African postcolonial female authors and their respective translations. Both novels are written in hybrid languages, African in their background and Europeanized in their form, namely: the French of Senegalese author Ken Bugul and the English of Nigerian author Buchi Emecheta. The departing point of this study is the premise established in postcolonial studies affirming that hybrid postcolonial texts have significantly specific language features and numerous cultural references (many of them in vernacular languages such as Wolof or lgbo) that represent a stumbling block to their translation practice. In our hypothesis, a single trend or strategy cannot be applied, but rather a balance between the different translational possibilities must be sought. From a womanist perspective, we will illustrate fragments of these works in order to analyse how the hybrid language and the African identity are exposed. Likewise, we will study the translational treatment that certain illustrative fragments of these African women's writings have experienced towards Spanish.
\end{abstract}

Key words: Translation; Hybridism; Postcolonial African Literature; Womanism; Cultural Specific Items (CSI); Identity.

\title{
1. INTRODUCCIÓN
}

Una de las principales características político-sociales del s. XX ha sido la descolonización del sur global, y, fruto de esta, los movimientos migratorios y la otredad (Gane 2003), particularidades que han ido transformando las identidades contemporáneas. Identidades que se reflejan, lógicamente, en la literatura, en lo que los estudios culturales han denominado el lenguaje híbrido del discurso literario poscolonial eurófono. De ahí el propósito de este trabajo: ofrecer evidencias de que, además de las particularidades lingüísticas propias inherentes a estos textos ${ }^{1}$, los culturemas ${ }^{2}$ identificativos

1. A propósito de las particularidades de este primer nivel textual lingüístico, imposibles de abarcar en el presente estudio, consultar Rodríguez-García Alba (2018)

2. Según la definición de Vlájov y Florin $(1969,438)$, los culturemas son «palabras (y locuciones) de la lengua popular que representan denominaciones de objetos, conceptos o fenómenos típicos de un ambiente geográfico, de una cultura, de la vida material o de las peculiaridades histórico-sociales de un pueblo, una nación, un país o una tribu: por eso, estas palabras son portadoras de un colorido nacional, local o histórico y no tienen correspondencia precisa en otras lenguas». Las denominaciones para los culturemas son diversas, a saber: realia (Escuela eslava y Escuela de Leipzig), palabras culturales (Newmark), nombres de referentes culturales específicos (Cartagena), referencias culturales (Escuela de Granada), segmentos marcados culturalmente (Mayoral y Muñoz), culturemas (Nord), léxico vinculado a otra cultura (Katan), etc., como

Isabel PASCUA FEBLES y Alba RODRÍGUEZ-GARCÍA Traducir las identidades africanas: una mirada mujerista
CLINA

vol. 5-1, June 2019, 95-112

elSSN: 2444-1961

Ediciones Universidad de Salamanca - CC BY-NC-ND 
de sus cosmovisiones están muy presentes en las escrituras poscoloniales africanas, y además de que han sido usados como señas de esas nuevas identidades desde un prisma mujerista, término acuñado por M. López (2003) como equivalente del inglés womanism. En concreto, nos referiremos a cómo las autoras poscoloniales Ken Bugul, de Senegal, y Buchi Emecheta, de Nigeria, han utilizado las lenguas coloniales y se han servido de numerosos culturemas relativos a las realidades naturales, materiales, socio-históricas y religioso-culturales de sus respectivas culturas para mostrar al mundo su identidad híbrida, femenina, euroafricana. A este fin, mostraremos ejemplos que confirmarán la hipótesis de que las referencias a su hibridismo y a su visión del mundo desde el prisma del género aparecen en los textos meta, aunque de diversa manera, dependiendo de la tendencia traductológica aplicada, e ilustraremos qué implicación ha supuesto su praxis traductológica para la comprensión del texto traducido por parte de los receptores meta.

Nos basamos en algunos de los más destacados y recientes estudios sobre la traducción postcolonial (Tymoczko 2006, Bandia 2008, Batchelor 2009 o Manfredi 2010, entre otros), los que afirman que esa otredad, ese third space híbrido (Bhabha 1994) debe ser respetado por quien afronta la traducción de ese texto peculiar. Para nuestro trabajo, hemos elegido un texto en francés y otro en inglés, ambos catalogados como escritura poscolonial femenina africana, empapados de referencias culturales africanas en wolof y en igbo, y sus respectivas traducciones a otra lengua europea, el español. Esta escritura ha sido, para muchos autores y autoras poscoloniales, una forma de presentar su cultura y lenguaje híbrido, su identidad entre fronteras, una necesidad de expresión de sus culturas e identidades propias. Hasta el punto de que algunos llegaron a convertirla en un «botín de guerra», como fue el caso radical del escritor keniata Ngugi wa Thiong'o, quien en un momento determinado decidió dejar de escribir en inglés, traducir sus novelas a las lenguas kikuyu y suajili ${ }^{3}$ o escribir directamente en estas lenguas como acto importante en su resistencia anti-colonialista. En su obra maestra Decolonising the Mind: the Politics of Language in African Literature (1986), este académico defendía la necesidad de descolonizar las prácticas lingüísticas impuestas por la colonización inglesa. En esta obra, insistía en "decolonize the mind», en usar las lenguas vernáculas africanas para reflejar las culturas africanas, la autoestima y la identidad cultural (Pascua 2018, 70). Al referirse a Ngugi wa Thiong'o, Bandia sostenía que «His writings in Kenyan languages are considered to be part and parcel of the anti-imperialist struggles of Kenyan and African peoples» (2006, 372).

señala Rodríguez-García (2015). En cuanto a su definición, la gran mayoría de los autores están de acuerdo con una definición común de los mismos, cercana a la previamente presentada.

3. La primera, lengua de la etnia mayoritaria en Kenia y la segunda, lengua ampliamente utilizada en Tanzania y en Kenia. Ambas catalogadas como lenguas bantú, pertenecientes a la familia congo-níger.

Isabel PASCUA FEBLES y Alba RODRÍGUEZ-GARCÍA Traducir las identidades africanas: una mirada mujerista
CLINA

vol. 5-1, June 2019, 95-112

elSSN: 2444-1961

Ediciones Universidad de Salamanca - CC BY-NC-ND 
Al tratar la colonización y descolonización, no debemos olvidar las cuestiones relativas al poder. Vidal $(2018,35-36)$ cita a la novelista nigeriana Chimamanda Ngozi Adichie, quien en su conferencia titulada "The Danger of a Single Story» (TEDGlobal, 2009) ${ }^{4}$ advierte de que es imposible hablar de una sola historia sin hablar de poder y señala que existe una palabra en la cultura igbo, nkali, que significa «ser más importante que otro", utilizada para subrayar el papel que desempeña el poder a la hora de decidir quién cuenta y cómo se cuenta una historia (Vidal 2018, 35).

Además de tratar nuestro trabajo desde la perspectiva de la traducción poscolonial, lo haremos también desde el womanism, con una mirada mujerista, ya que abordamos en particular la literatura femenina. La segunda generación de escritoras a la que pertenecen nuestras autoras guarda, sin duda, relación con sus predecesoras, pero anuncian ya un cambio en la voz del «yo» femenino, que plantea abiertamente una denuncia de la situación de las mujeres en el continente africano. Man $(2007,11)$ afirma al respecto que, tras una primera generación fundamentalmente autobiográfica en donde fueron expuestos los males particulares de las mujeres, una segunda generación aparece y toma consciencia de que hay que cambiar toda la sociedad. Esto hace que todos sus escritos, no solo los relativos a las mujeres sino también a la política, la economía, la sociedad, a Occidente, etc., estén marcados por esta visión mujerista de la que nos ocupamos. Entre otros autores, Díaz (2007, 97-98) considera la escritura femenina como un arma de protesta, un elemento de denuncia para transformar la sociedad; sus objetivos van más allá del feminismo occidental. Y, desde este punto de vista, debemos señalar la actitud de ciertas escritoras africanas al plantear en sus obras sus reivindicaciones comunitarias y realistas. Se centran en los problemas y experiencias de la vida cotidiana, en su deseo de crear un ambiente igualitario de clases, razas, género; de llegar a la reconciliación entre todos los miembros de la comunidad global. Lucha que va más allá del frente que defiende el feminismo occidental, que bajo la mirada y experiencia de estas autoras africanas está más centrado en el género, es más eurocéntrico, individualista y no las tiene del todo en cuenta de alguna manera, pues las realidades propias son más complejas. En principio, como ciudadanas de un mundo oprimido por la raza y por la clase social, estas mujeres no encontraban un sitio apropiado con el feminismo occidental, pero necesitaban hacerse visibles. Con esa mirada womanista/mujerista, toman en consideración no solo la desigualdad de género, sino la opresión del racismo y clasismo; la experiencia de sus mujeres; su cultura, sus mitos y su vida espiritual (Pascua 2017). Así, muchas consideran que al encontrarse ante distintos frentes, la mujer negra africana ha relegado, de alguna forma, su condición fundamental como mujer. Quizá «las voces de las mujeres africanas hayan sido acalladas en su reivindicación por el estatus de igualdad con los hombres» (López 2003, 157). Esos

4. Publicada en español bajo el título El peligro de la historia única (Literatura Random House, 2018).

Isabel PASCUA FEBLES y Alba RODRÍGUEZ-GARCÍA Traducir las identidades africanas: una mirada mujerista
CLINA

vol. 5-1, June 2019, 95-112

elSSN: 2444-1961

Ediciones Universidad de Salamanca - CC BY-NC-ND 
diferentes frentes han hecho que muchos se pregunten qué es lo que viene primero, si la lucha por la igualdad o la lucha contra el imperialismo cultural occidental. Quizá la respuesta sea que son inseparables: «No one without the other» (Sougou 2002, 21).

Las autoras de las dos obras aquí analizadas, así como muchas de las autoras africanas, ven el mundo, lo comentan y lo critican desde la interseccionalidad, concepto que se refiere a la lucha de las mujeres en la que convergen varios frentes simultáneos, y que no se limitan al género, sino que a este se le suman las discriminaciones relativas a su raza, a su sexualidad y su clase social. Crenshaw (1989) lo utilizó para referirse a la situación de intersección entre sexismo y racismo sufrido por las feministas afroamericanas, y hoy en día este término y esta visión del mundo se ha convertido en uno de los conceptos clave alrededor de los que se desarrollan los estudios y escritos sobre feminismo. Las escritoras africanas, consciente o inconscientemente, están inmersas en esta situación y por lo tanto escriben y describen el mundo desde este prisma, característica que no debe ser dejada de lado en el proceso traductológico, sino que, al contrario, debe ser manifestada consecuentemente en términos lingüísticos y extralingüísticos.

\section{CONTEXTUALIZACIÓN}

Para entender la literatura africana poscolonial es imprescindible tener en cuenta la particular situación política, social y económica de aquellos países africanos recién creados e independientes de sus respectivas colonias a partir de la segunda mitad del s. XX. La historia político-cultural del continente tuvo gran influencia en la escritura de sus literaturas, que se desarrollaron en el marco de fronteras culturales difusas y en las que los autores se encontraron en tierra de nadie. Una literatura donde lo local del continente se entrelaza y se confunde con lo global del mundo, dando lugar así a una escritura y, consiguientemente, a una práctica traductológica que necesita de un tratamiento glocal ${ }^{5}$ (Rodríguez-García 2015, 330).

Como ya adelantamos, ejemplificaremos nuestro trabajo con fragmentos de las obras de dos importantes autoras africanas eurófonas, pertenecientes a la ya

5. El término glocal entendido como la simultaneidad o co-presencia de tendencias universalizadoras y particulares (Robertson, 2003). Por ende, un tratamiento glocal consistiría en un equilibro, y una glocalización de la traducción sería, según defiende Rodríguez-García (2019): «a more comprehensive model, which will combine some tenets of the classical Western procedures, mixed strategies a priori opposite from Schleiermacher's dichotomy, and different aspects from the interdisciplinary fields of postcolonial and cultural studies. All this aims to avoid Eurocentric praxis, reductionist approaches, excessively domestic or exotic re-expressions and fake representations of the source texts we are dealing with. The final objective is to achieve faithful and culturally respectful literary target texts».

Isabel PASCUA FEBLES y Alba RODRÍGUEZ-GARCÍA Traducir las identidades africanas: una mirada mujerista
CLINA

vol. 5-1, June 2019, 95-112

elSSN: 2444-1961

Ediciones Universidad de Salamanca - CC BY-NC-ND 
catalogada «segunda generación» de escritoras del continente, que contienen referencias culturales propias y que transmiten los sentimientos de mujerismo. Por una parte, la nigeriana Buchi Emecheta, inmigrante en Gran Bretaña e icono de la literatura nigeriana Black British o afrobritánica, cuyas obras son un reflejo de la múltiple opresión de la mujer africana en las que reclama su hibridez e identidad. Por otra parte, la senegalesa Ken Bugul (Mariétou Mbaye Mbiléoma), conocida defensora de los problemas de las mujeres africanas, ganadora entre otros del Grand Prix Littéraire de l'Afrique Noire en el año 2000, y feroz ilustradora de cómo la cultura, la tradición y la religión influyen y conforman lo femenino más allá del concepto de feminismo occidental.

Ambas autoras escriben desde el mujerismo y señalan la múltiple opresión desde distintos frentes sobre la mujer negra africana. Para Boyce Davies (López 2003, 145) las características en esa escritura africana serían: el colonialismo, la inmigración, el racismo, su propia condición de mujer, el contemplar la historia con recelo pues la tradición y el colonialismo pesan demasiado, respetar la maternidad pero oponerse si es obligatoria, aceptar la poligamia, pero cuestionando el privilegio marital del hombre, etc.

Para analizar y entender mejor cómo muestran la identidad a través de ese lenguaje híbrido, analizaremos la obra Riwan ou le chemin de sable, que fue publicada en 1999 y conforma el cierre de lo que ha venido en denominarse la trilogía autobiográfica de Ken Bugul ${ }^{6}$. En esta tercera obra relata su vuelta a Senegal tras su periplo europeo, su reconciliación social e histórica con su país, y su curación vital y mental gracias a haberse convertido libre y voluntariamente en la vigésima octava mujer de un líder espiritual islámico. Una obra que gira en torno a tres mujeres y a las vivencias y los diversos destinos de estas, repleta de referencias culturales cruzadas, de culturemas en lengua wolof y de conceptos o visiones no siempre fáciles de descifrar por los lectores, que examinaremos a la luz de su traducción Riwan o el camino de la arena, llevada a cabo por Nuria Víver Barri en 2005. De la nigeriana Buchi Emecheta, escogimos la obra The Joys of Motherhood (1979), que escribió como inmigrante en Gran Bretaña. El título lo tomó del mensaje de la escritora Flora Nwapa7, en su novela Efuru. La novela de Emecheta contiene numerosas referencias en lengua igbo, lengua tonal que usa el alfabeto latino y que hablan 18 millones de personas de la etnia igbo, de la zona conocida como Biafra. Y nos centramos en su traducción, Las delicias de la maternidad, realizada en 2004. En el original en lengua inglesa no se explican las referencias culturales en lengua igbo y no es fácil comprender la intención de Emecheta.

6. A saber: Le baobab fou (Présence Africaine,1982), Cendres et braises (L'Harmattan, 1994) y Riwan ou le chemin de sable (Présence Africaine, 1999). A estas tres le han seguido más de una decena de novelas.

7. También nigeriana y perteneciente a la conocida como primera generación de escritoras, a la que también pertenecen Mavel Dove Danquah, la keniata Grace Ogot, la sudafricana Noni Javabu o la ganesa Ama Ata Aidoo.

Isabel PASCUA FEBLES y Alba RODRÍGUEZ-GARCIA Traducir las identidades africanas: una mirada mujerista
CLINA

vol. 5-1, June 2019, 95-112

elSSN: 2444-1961

Ediciones Universidad de Salamanca - CC BY-NC-ND 
Los británicos y los franceses han tenido contacto con pueblos africanos durante sus períodos coloniales y poscoloniales y, debido a este contacto entre las metrópolis y las colonias, es posible que los lectores de los textos originales (TO) conocieran algunos de los términos y realidades culturales expuestos en ellos. Quizá el deseo de las autoras fue por momentos enfrentar a sus lectores a vocablos y realidades que, si bien en ocasiones pudieran ser desconocidos, reflejaban la cultura y la cosmovisión euroafricana de estas autoras y sus orígenes. En el primero de los casos, muchos de los culturemas de Ken Bugul pasan desapercibidos o son difícilmente comprensibles para los lectores del texto meta (TM), pues quedan en su mayor parte libres de explicación, adaptación o aclaración alguna por parte de la traductora. En el segundo caso, la traductora de Buchi Emecheta, sin embargo, recurrió en algunas ocasiones a la explicación dentro del TM y añadió 16 notas de traducción a pie de página, que implicaron amplificación en este.

\section{TRADUCIR LAS REFERENCIAS CULTURALES}

En el análisis comparativo-contrastivo de los textos nos guiaremos, entre otros, por los estudios sobre los culturemas o referentes culturales de las funcionalistas Nord (1997) y Witte (2017), entendidos como fenómenos sociales relevantes para los miembros de una cultura que, al compararlos y contrastarlos con los de otra cultura, se aprecia y comprende mejor la especificidad. A la vez, tomamos el concepto de referencia cultural de Mayoral (1994, 73-78), como elemento del discurso, la expresión lingüística que refleja una forma de pensar, de ver la realidad y de actuar, condicionados por la cultura de la que formamos parte.

Se trata de referencias a lugares físicos o naturales, objetos materiales, nombres propios, comportamientos y valores, creencias religiosas, realidades histórico-sociales, etc. Para analizarlos, nos servimos de la clasificación propuesta por Rodríguez-García $(2015)^{8}$. En definitiva, analizamos algunos de los elementos aparecidos en los TO que podrán ser compartidos o no por las dos culturas y que suponen una marca de diferencia cultural entre ellas. La producción desde la compleja relación entre el TO, otros textos, los lectores y el contexto cultural se entenderá como intertextualidad cultural.

Evidentemente, la traducción presupone elecciones, alternativas, estrategias y decisiones y, por tanto, el traductor debe estar preparado, tener una gran competencia cultural, reconocer y comprender esos culturemas y decidir las estrategias de

8. En su investigación, defendida como tesis doctoral (Universidad de Las Palmas de Gran Canaria, 2015), los agrupa en una taxonomía cuyo criterio es la referencia cultural a la que señalan, a saber: realidad natural, realidad material, realidad socio-histórica y realidad religiosocultural. Consultable en http://hdl.handle.net/10553/21688

Isabel PASCUA FEBLES y Alba RODRÍGUEZ-GARCÍA Traducir las identidades africanas: una mirada mujerista
CLINA

vol. 5-1, June 2019, 95-112

elSSN: 2444-1961

Ediciones Universidad de Salamanca - CC BY-NC-ND 
traducción que debe usar en aras de lograr un TM que tenga sentido para sus lectores, al tiempo que respete la particular idiosincrasia del TO africano y de su sustrato. En palabras de Vidal «el traductor asegura la supervivencia del original, donde original no significa más que lo que viene antes, lo que inicia, desencadena o libera una nueva palabra» (2007, 108); es esa nueva palabra, cargada de hibridismo y de otredad en sus sentidos más positivos, la que hay que lograr en la particular práctica traductológica de los textos que nos ocupan. Estamos de acuerdo con la consideración de Gyasi (1999, 82), quien sostiene que «if the translation of African literature from African languages into European languages is no easy task, the translation of this literature from one European language into another presents even more problems». Por ello, en el análisis de las referencias culturales de las novelas híbridas citadas y de las tendencias y estrategias que se utilizaron al traducir, nos reafirmamos en que no hay ninguna que, «utilizada strictu sensum, logre por si sola hacer frente a los escollos que estos textos presentan» (Rodríguez-García 2019). Partimos de la conocida dicotomía de Venuti (1995), que distingue entre extranjerización (conservar referencias propias de la cultura del TO en el TM, rompiendo si es necesario las convenciones del TM, para asegurar que no se pierde el significado, donde se destaca lo extraño, lo diferente y desconocido) y domesticación (traer las referencias del TO hacia la cultura del TM). Venuti, al igual que muchos otros estudiosos, enfatiza la extranjerización, pues considera que al domesticar se borran los valores culturales del TO. Sin embargo, y aunque estemos absolutamente de acuerdo en la conveniencia de preservar las referencias culturales por razones, entre otras, deontológicas, no pensamos que la extranjerización sea la única vía, pues hemos podido observar y comprobar que, en ocasiones, es la domesticación la que sirve de puente y garantiza la comprensión del texto y el entendimiento de identidad cultural del TO por parte de los lectores del TM. Por tanto, cuando las traductoras mencionadas optan por una u otra, muestran, por una parte, la tendencia traductológica de la misma, y, por otra, el grado de tolerancia cultural que estas consideran que los lectores del TM tienen hacia el TO.

\section{ANÁLISIS COMPARATIVO DE LAS OBRAS}

Vayamos, pues, al análisis de algunos ejemplos de fragmentos de los TO ilustrativos de lo explicado. Mostraremos culturemas relativos a los nombres propios, lugares, referencias históricas y sociales, religiosas y culturales, expresiones, la consideración femenina, etc., para mostrar así cómo a través de esta escritura, nuestras protagonistas presentan su cultura, su lenguaje híbrido, su identidad africana y visión femenina de la realidad.

Isabel PASCUA FEBLES y Alba RODRÍGUEZ-GARCÍA

Traducir las identidades africanas: una mirada mujerista
CLINA

vol. 5-1, June 2019, 95-112

elSSN: 2444-1961

Ediciones Universidad de Salamanca - CC BY-NC-ND 


\subsection{Riwan ou le chemin de sable, de Ken Bugul}

En primer lugar, anotaremos aquí fragmentos del TO acompañados de sus correspondientes en el TM y agrupados en función de la taxonomía antes citada, lo que nos permitirá, en un primer tiempo, mostrar el discurso híbrido de la condición femenina e identitaria de la(s) mujer(es) africana(s) y su traducción. A continuación, explicaremos brevemente estos fragmentos para, por una parte, acceder a una mejor comprensión de las referencias culturales aparecidas en el TO a propósito del hibridismo y del fenómeno identitario femenino objeto de este estudio; y, por otra, para poder llevar a cabo un análisis de la praxis traductológica que estos han sufrido y proponer soluciones alternativas para beneficio de los lectores del TM. Los ejemplos aquí presentados contienen claras referencias a los usos, los estereotipos, los comportamientos, las tradiciones, las exigencias y costumbres de las mujeres africanas, dando así cuenta de la importancia que la autora consagra a esta identidad y a los patrones de comportamiento social (impuestos en algunos casos, pero también elegidos en tantos otros) por parte de ellas en sus respectivas sociedades.

TO: Ces femmes de Lomé ou de Cotonou pouvaient acheter un homme (p. 185).

TO: Quand la mauresque avait fait le tour de ses maisons de prédilection (p. 145).
TM: Las mujeres de Lomé o de Cotonou podían adquirir un hombre (p. 211).

TM: Después de que la morisca recorriera sus casas de predilección (p. 169).

Tabla 1: Realidad natural (geografía africana)

En el primer caso, Lomé y Cotonú como referencias al empoderamiento y la fuerza de las togolesas y beninesas en sus respectivas sociedades y, en el segundo, «morisca» (mora) en referencia a la estereotipada imagen, en ocasiones no positiva, de las africanas del norte y del Magreb. Para colmar, por una parte, el «vacío» geográfico, y por otro, la "carga estereotipada», proponemos como fragmentos meta: «Las togolesas de Lomé o las beninesas de Cotonú podían comprarse un hombre», y "Después de que la mora se diese una vuelta por sus casas preferidas».

TO: Ses ensembles xit mbal, taille basse et pagne, ses ensembles petit boubou, ses mouchoirs bien pliés (p. 84).

TO: Hidroquinone-Mercure et autres saloperies. Devenir claires. Devenir blanches (p. 96).
TM: Sus enseres xit mbal, blusón y pareo, sus conjuntos de bubús de diario (p. 104).

TM: Hidroquinona-mercurio y otras porquerías. Volverse claras. Volverse blancas (p. 116).

Tabla 2: Realidad material (vestimentas y enseres, objetos, productos...)

En esta ocasión observamos fragmentos que hacen referencia a la vestimenta de las senegalesas y a la importancia que estas dan a su imagen (muy reputadas en África

Isabel PASCUA FEBLES y Alba RODRÍGUEZ-GARCÍA Traducir las identidades africanas: una mirada mujerista 
por su coquetería y belleza) y a la crítica por parte de la autora de esas pócimas mágicas tan utilizadas por sus compañeras para aclararse la piel y encajar en el canon de belleza occidental. Respecto al primer ejemplo, y con el fin de mantener las realidades materiales sin «adaptarlas» al español y colmar la omisión que comporta el TM, proponemos: «Sus conjuntos xit-mbal, taille basse y pagne, sus bubús de diario, sus pañuelos bien doblados». Para el segundo de los ejemplos, y con el fin de ilustrar la «epidemia psicológica» que suponen los tratamientos dermatológicos para el blanqueamiento de la piel en las africanas, proponemos: «Untarse hidroquinona, mercurio y demás porquerías. Para aclararse la piel. Para convertirse en blancas».

TO: Celles qu'on disait allées à l'école des Autres (p. 35).

TM: Que habían ido a la escuela de los Otros (p. 54).

TO: Ce n'était pas la fille de la Badiène ou du Nidjiay (p. 44).

TM: No era hija de la badiène o del nidiay (p. 64).

Tabla 3: Realidad socio-histórica (educación colonial, jerarquías familiares...)

En este tercer caso, vemos cómo se señala una clara diferencia entre aquellas mujeres instruidas en la escuela colonial (la de los Otros) y las que son fruto del sistema senegalés. Sin nociones históricas sobre el pasado colonial de Senegal se hace complicado entender a quién se refiere «otros» en el TM. Así, proponemos «Que habían ido a la escuela de los Blancos» como alternativa de traducción. En el segundo ejemplo observamos cómo, a través de dos conceptos clave de la cultura y lengua wolof, se marca la importancia del vínculo familiar que concede ser hija de una tía paterna (bàjjen) o de un tío materno (nijaay) por encima de cualquier otro lazo familiar. Con la voluntad de no invisibilizar la lengua y la cultura origen, proponemos para este fragmento: «No era la hija de la Bàjjen ni del Nijaay», atendiendo a la transcripción oficial wolof del reputado lingüista Diouf (2003).

TO: Cette veuve d'un grand serigne ne pouvait être que la takko* d'un autre grand Serigne (p. 104).

TO: Sinon comment la plupart des prophètes auraient-ils pu accomplir leur mission sans l'assistance de femmes riches de ces époques là? Xadija! Merci! (p. 94).
TM: La viuda de un gran Seriñe solo podía ser la takko* de otro gran Seriñe (p. 125).

TM: Si no, ¿cómo habrían podido realizar su misión la mayoría de los profetas sin la ayuda de las mujeres ricas de su época? ¡Xadija! ¡Gracias! (p. 114).

Tabla 4: Realidad religioso-cultural (tradición e historia religiosa)

Por último, ilustramos la referencia a una costumbre socio-religiosa según la cual la viuda de un seríñ (líder espiritual) solo puede convertirse en la esclava (takko) de uno de sus homólogos, tal como dicta la sociedad patriarcal. Proponemos para el TM el término 
wolof serín por ser, además de correcto, fonéticamente más cercano al original que su transcripción francesa «serigne». Así, tendríamos como alternativa: «Esta viuda de un gran serín no podía ser sino la takko de otro gran seríñ». En el segundo ejemplo observamos la importancia capital de las mujeres en las religiones, a través del apunte sobre las riquezas de la mujer del profeta que permitieron, entre otros, la misión de difusión y propagación del Islam de su marido Mahoma. Proponemos para este fragmento: «¿Cómo podría entonces haber cumplido su misión la mayoría de los profetas si no hubiese sido por la ayuda de mujeres ricas de aquellas épocas? ¡Gracias, Jadiya!».

Dando visibilidad a estas identidades a través de estos segmentos marcados culturalmente, la autora logra empoderar y dar a conocer al mundo estas identidades femeninas a través de su escritura. En los casos aquí señalados, se puede comprobar cómo los fragmentos no han sufrido tratamiento alguno en el TM, más allá de haber sido trasladados tal cual aparecen en el TO. A nuestro juicio, esto conduce a una cierta pérdida de información de vital importancia para los lectores del TM. Por ello defendemos que, a través de diferentes estrategias traductológicas, fruto de un tratamiento equilibrado entre extranjerización y domesticación (paráfrasis, transcripción fonética del wolof en español, uso de la cursiva para ciertos términos, etc.), podría haberse producido un resultado más acorde con la voluntad de difusión de la identidad femenina africana por parte de la autora, y un texto más comprensible para los lectores del TM. Por ello, junto con el muestrario y su comentario crítico, se han querido proponer, de manera ilustrativa, alternativas de traducción posibles en consonancia con nuestra voluntad de glocalización de la práctica traductológica.

Concluiremos este apartando con un dato a tener en cuenta: en un análisis previo de esta obra llevado a cabo en el año 2015, con un prisma más general y no únicamente centrado en las realidades femeninas, identificamos más de un centenar de fragmentos textuales híbridos. De entre estos, un total de 70 fueron seleccionados por su alto interés para el objeto de nuestro estudio. Tras los resultados del exhaustivo análisis, concluimos que un $16 \%$ correspondían a la realidad natural, un $25 \%$ a la material, un $22 \%$ a la socio-histórica y un $31 \%$ a la religioso-cultural, completados por un $6 \%$ de omisiones en el TM. Esta distribución porcentual da cuenta de la diferencia cultural entre los TO y TM estudiados, que se manifiesta en diversos grados, siendo las realidades religioso-culturales las más distantes.

\subsection{The Joys of Motherhood, de Buchi Emecheta}

En el análisis de la novela de Buchi Emecheta escrita en inglés, comprobamos una gran fuerza identitaria. La autora utilizó más de 200 referentes culturales como signos de su identidad africana, muchas fueron preservadas por la traductora con el mismo propósito.

Isabel PASCUA FEBLES y Alba RODRÍGUEZ-GARCÍA Traducir las identidades africanas: una mirada mujerista 
Señalaremos solo los ejemplos más significativos, y las Notas de Traducción:

Nombres propios: los 79 nombres (43 antropónimos y 32 topónimos) se preservaron como préstamos, incluso los más difíciles de pronunciar.

Antropónimos: Nnu Ego, personaje principal, Nwokocha Agdabi, Oshiaju, Nnaife, Omunna, Nwakusor, Obi Idayi, Folorunsho, etc.

Tabla 1:

TO: Mama Oshia, customary manner for a woman with a male child (p.115).
TM: Mamá Oshia, saludo que se utiliza para una mujer que tiene un hijo varón (p.156).

Topónimos: Ibuza; Ogboli; Asaba; Emekuku Owerri; Akinwunmi Street; Excepciones: Fernando Po/Fernando Poo, Amelika/América.

- Realidad material (vivienda):

Tabla 2:

TO: homestead (p. 9). TM: recinto (p. 30).

(N. de la T.): «Del inglés de África Occidental y Sudáfrica, traducida en este texto unas veces como «recinto" o «concesión» (en referencia a pueblo) y otras como "casa", (en alusión a una casa moderna de la ciudad); se refiere al espacio físico habitado por una comunidad. En el primer caso se trataría de la familia tradicional compuesta por el cabeza de familia y sus esposas, donde el padre tiene su propia cabaña y cada una de las esposas tiene una propia donde vive con los hijos que ha tenido; sin embargo, en la ciudad, se trata de un edificio en el que cada familia solía alquilar una sola habitación en la época colonial, algo que sigue sucediendo entre las familias pobres en la actualidad».

(Indumentaria 7): Todos préstamos puros; de ellos 3 con Notas de traducción. En el ejemplo, no se aclara en el TM, pero queda claro por el contexto: tela unas veces falda y otras vestido.

Tabla 3:

TO: lappa (p.7). TM: lappa (p. 28).

- Realidad socio-histórica y realidad religioso-cultural. Los 75 culturemas encontrados, a nuestro entender, son las referencias que mejor reflejan la identidad africana en la obra de Emecheta. Hemos agrupado las más importantes en 
diferentes temas: realidad religiosa; el colonialismo; la realidad social; el papel de la mujer y la nueva mujer africana.

- Realidad religiosa (10):

Tabla 4:

TO: Mammy Waater (p. 44). TM: Mammy Waater (p. 74).

(N.de la T.): «Diosa de las aguas, como se le denomina en el inglés de África Occidental y del inglés pidgin hablado en toda la costa del Golfo de Guinea, incluyendo Guinea Ecuatorial. En el período colonial se la representa como una diosa mestiza con una abundante melena ondulada, que podría interpretarse como un sincretismo entre la imagen de la mujer blanca colonial y la diosa acuática tradicional presente en varias religiones de la región, como la igbo (donde esta diosa recibe el nombre de Uhamiri) o la yoruba (cuyo nombre es Yemanya)».

- Realidad social:

Tabla 5: Las esclavas

TO: A good slave was supposed to jump into the grave willingly, happy to accompany her mistress (p. 20).
TM: Se suponía que una buena esclava debía saltar a la sepultura por su propia voluntad, contenta por acompañar a su señora (p. 43),

Tabla 6: Celebración de boda

She went with seven hefty men and seven young girls carrying her possessions. There were seven goats, baskets of yam, yards and yards of white man's clothes, twenty four home-spun lappas, rows and rows of Hausa trinkets and coral beads [...] (p. 28).
Envió a su hija con siete hombres robustos y siete jovencitas que le llevaban sus pertenencias. Había siete cabras, cestas de ñame, y metros y metros de tela del hombre blanco, venticuatro lappas tejidas en el pueblo, innumerables fruslerías y cuentas de coral (p. 55).

Tabla 7. El colonialismo:

TO: Are we not all slaves to the white men, in a way? (p. 130).

TM: ¿No somos todos esclavos de los blancos, de alguna manera? (p. 175).

TO: ...। can't speak that stuff they call "canary» English [...]

TM: Yo no sé hablar esa cosa que llaman inglés, el inglés de los canarios.

«Pidgin English, old boy, pidgin English» (p. 103).

- Se llama pidgin, querido muchacho, es el inglés pidgin (p. 144).

(N. de la T.): «Alusión al inglés pidgin, denominado despectivamente parrot English (inglés de loros) o canary English por los británicos en las áreas colonizadas donde escuchaban esta lingua franca entre la población autóctona». 
Tabla 8: El papel de la mujer

TO: A woman without a child for her husband was a failed woman (p. 65).

TM: Una mujer sin un hijo que ofrecer a su marido era una mujer fracasada (p. 100).

TO: Don't you know that according to the customs of our people you, Adaku, the daughter of whoever you are, are committing an unforgivable sin [...] I know you have children, but they are girls (p. 185).

TM: ¿No sabes que según las costumbres de nuestro pueblo tú, Adaku, la hija de quien seas, estás cometiendo un pecado imperdonable? [...] Sé que tienes hijos, pero son niñas (p. 247).

Tabla 9:

TO: She belonged to both men, her father and her husband, and lastly to her sons ( $p$. 207).

TM: Pertenecía a los dos hombres, a su padre y a su marido, y en último lugar a sus hijos varones (p. 274).

Tabla 10: La nueva mujer

TO: God, when will you create a woman who will be fulfilled in herself, a full human being, not anybody's appendage? (p. 209).

.TO: Nnu Ego had it all, yet still did not answer prayers for children (p. 254).

TM: Dios mío ¿cuándo vas a crear a una mujer que se realice por sí misma, un ser humano completo, no el apéndice de otro? (p. 277).

TM: Nnu Ego lo tuvo todo, pero sin embargo nunca respondió a las oraciones de quienes le pedían hijos (p. 333)

Nnu Ego, después de entregar su vida a su esposo y mantener a sus nueve hijos, murió sola, sin hacer ruido, sin un hijo o una amiga que le diera la mano. Tras la dura lucha de la vida, ¿tuvo alguna recompensa? No, estuvo demasiado ocupada buscando las delicias de la maternidad'

\section{CONCLUSIÓN}

Desde nuestra mirada mujerista, nos encontramos con dos autoras cuyos textos ilustran a la perfección lo que se ha venido a llamar la literatura poscolonial femenina eurófona. Por su parte, Buchi Emecheta, con su lenguaje desafiante y nada inocente, patente incluso en la elección del nombre de la protagonista (Boehmer 2008): Nnu Ego (new ego/la nueva yo), reivindica no solo su identidad africana sino el womanism, donde cuestiona el colonialismo, la maternidad, el matrimonio, la poligamia, las tradiciones a ultranza y también la desigualdad de género. Por su parte, Ken Bugul sigue la misma línea de esta y otras autoras representantes de la segunda generación de escritoras africanas.

\section{Interpretación personal inspirada en el final de la historia de la protagonista.}

Isabel PASCUA FEBLES y Alba RODRÍGUEZ-GARCIA Traducir las identidades africanas: una mirada mujerista 
Sin ir más lejos, su propio «nombre» literario es un pseudónimo que en la lengua wolof quiere decir «la que nadie quiere», en gran parte por su experiencia de rechazo identitario, tanto al llegar a Europa como a su vuelta a su Senegal natal, y por el abandono materno que sufrió cuando era niña. Además de estas razones, la primera obra de Ken Bugul (Le baobab fou, 1984) fue considerada tan atrevida y chocante por su editor, que le recomendó utilizar un pseudónimo para evitar problemas en su Senegal natal debido al controvertido contenido de esta obra, y de las que siguieron. En sus novelas, la autora critica los patrones africanos, pero también los occidentales, a los que las mujeres del continente y de la diáspora se ven sometidas y se refiere con frecuencia a sus congéneres, empoderando y avivando así sus luchas. Habla sin tapujos de tabúes, como la homosexualidad, las drogas o el alcohol y defiende la libertad por encima de todo, lleva al feminismo y la libertad que este debería defender a límites insospechados, como el de ser libre para convertirse en la vigésimo octava mujer de un líder espiritual por elección propia. Ambas autoras exponen las realidades de las mujeres africanas poscoloniales y de su situación en el mundo, y dejan traslucir en sus novelas la fuerza de las identidades femeninas cruzadas, híbridas y en continua negociación que su particular historia ha dejado a su paso.

Concluimos que las literaturas poscoloniales africanas plantean retos específicos derivados de sus particularidades lingüísticas y extralingüísticas, retos que todavía están por abordar y a los que habría que dar solución a través de estudios concretos sobre su novelística, como el que hemos querido ilustrar en este trabajo. Además, en el caso particular de la escritura femenina, la identidad híbrida, la imagen de la mujer y la compleja situación que supone el género cobran una importancia textual sin precedentes, de ahí que deba ser llevado a cabo un tratamiento cuidadoso en este sentido. Los culturemas que empapan esta escritura tienen, en su mayor parte, una relación estrecha con estos condicionantes que son mostrados deliberadamente por sus autoras y son convertidos en escaparate y contexto de su particular construcción identitaria. La praxis traductológica se torna pues compleja, y, desde nuestro punto de vista, una única tendencia o estrategias reductoras no parecen darnos las claves para la solución, por lo que sugerimos que, para el éxito de los TM en lengua española, debe conseguirse cierto equilibrio entre ambas a través de un método glocalizado. O quizá recurrir al translanguaging (acto basado en un único repertorio obtenido a partir de varias lenguas) como sugiere Karen Bennett (2018) según lo postulado por García y Wei (2014). Por tanto, consideramos que ante un texto híbrido, debe aplicarse un método híbrido.

Los ejemplos de las traducciones de los textos expuestos muestran dos tendencias relativamente opuestas. El caso de la traducción de Buchi Emecheta tiende a la domesticación -16 notas de la traductora y algunas explicaciones- y se acerca a la traducción híbrida; sin embargo, el caso de Ken Bugul es más asimilable a la extranjerización. Ninguna de ellas sería exitosa si se dejara de lado completamente a la otra.

Isabel PASCUA FEBLES y Alba RODRÍGUEZ-GARCÍA Traducir las identidades africanas: una mirada mujerista 
Explicar excesivamente todos los elementos del TO hace que de alguna manera este pierda su color local y propio. Por otra parte, no explicar o aclarar ninguno de estos elementos propios hace que mucho de su contenido se pierda al volcarlo al TM.

Por todas estas razones concluimos que un tratamiento híbrido y glocal debe ser el marco en el que estos textos sean tratados en su praxis traductológica.

\section{BIBLIOGRAFÍA}

ACHEBE, Chinua. 1965/1997. "English and the African Writer». En Transition 75/76, The Anniversary Issue: SELECTIONS from Transition 1961-1976: 342-349.

ADICHIE, Chimamanda Ngozi. 2018. El peligro de la historia única. Barcelona: Literatura Random House. Traducido por Cruz Rodríguez Juiz.

BANDIA, Paul. 2006. "African Europhone Literature and Writing as Translation». En Translating Others (vol. II), ed. por Theo Hermans. Manchester: St. Jerome Publishing, 349-64.

BANDIA, Paul. 2008. Translation as reparation: Writing and Translation in Postcolonial Africa. New York: Routledge.

BATCHELOR, Kathryn. 2009. Decolonizing Translation: Francophone African Novels in English Translation. Manchester: St. Jerome Pub.

BENNET, Karen. 2018. «Traducción, migraciones y asimetrías en el mundo global» (Mesa redonda que tuvo lugar en el 1er congreso internacional "Traducción y sostenibilidad cultural: sustrato, fundamentos y aplicaciones» celebrado en la Universidad de Salamanca, noviembre 2018).

BHABHA, Homi. 1994. The Location of Culture. London: Routledge.

BOEHMER, Elleke. 2008. «Introduction» The Joys of Motherhood. Harlow, Essex: AWS Heinemann, i-vii.

BUGUL, Ken. 1982. Le baobab fou. Dakar: Nouvelles éditions africaines.

BuGUL, Ken. 1994. Cendres et braises. Paris: Editions L'Harmattan.

BuGUL, Ken. 1999. Riwan ou le chemin de sable. Paris: Présence africaine.

BuGUL, Ken. 2005. Riwan o el camino de arena. Madrid: Ed. Zanzíbar. Traducido por Nuria Víver Barri.

CRENSHAW, Kimberle. 1989. "Demarginalizing the Intersection of Race and Sex: A Black Feminist Critique of Antidiscrimination Doctrine, Feminist Theory and Antiracist Politics». En University of Chicago Legal Forum: 1989 (1): 139-167. Disponible en https://chicagounbound. uchicago.edu/cgi/viewcontent.cgi?article $=1052 \&$ context=uclf

DíAZ NARBONA, Inmaculada. 2007. Literaturas del África subsahariana y del Océano Índico. Cádiz: Universidad de Cádiz.

DIOUF, Jean Léopold. 2003. Dictionnaire wolof-français et français-wolof. Paris: Éditions KARTHALA Editions, 2003.

ECHERU, Michael J. 1998. Igbo-English Dictionary. New Haven: Yale University Press.

EMECHETA, Buchi. 2004. Las delicias de la maternidad. Madrid: Ed. Zanzíbar. Traducido por Maya García Vinuesa.

EMECHETA, Buchi. 2008. The Joys of Motherhood. Harlow, Essex: AWS Heinemann.

Isabel PASCUA FEBLES y Alba RODRÍGUEZ-GARCIA

Traducir las identidades africanas: una mirada mujerista 
GANE, Gillian. 2003. «Achebe, Soyinka, and Other-Languagedness». En The Creative Circle: Artist, Critic, and Translator in African Literature. African Literature Association Annual Series, ed. por Angelina E. Overvold, Richard K. Priebe y Louis Tremaine. Trenton NJ: Africa World Press: 131-149.

GARCÍA, Ofelia y Li WEl. 2014. Translanguaging: Language, bilingualism and education. London: Palgrave Macmillan

GYASI, Kwaku. 1999. «Writing as Translation: African Literature and the Challenges of Translation». En Research in African Literatures 30 (2). Bloomington: Indiana University Press: 75-87.

LÓPEZ RODRÍGUEZ, Marta Sofía. 2003. "Literatura africana feminista: una perspectiva mujerista». En Cuadernos Centro de Estudios Africanos, 3: 137-190.

MAN, Michel. 2007. La folie, le mal de l'Afrique postcoloniale dans Le baobab fou et La folie et la mort de Ken Bugul. Tesis doctoral. Columbia: University of Missouri. Disponible en https:// mospace.umsystem.edu/xmlui/bitstream/han- dle/10355/4794/research.pdf?sequence=3

MANFEDI, Marina. 2010. «Preserving Linguistic and Cultural Diversity in and through Translation: From Theory to Practice». En Mutatis Mutandis. Revista Latinoamericana de Traducción 3 (1): 45-72.

MAYORAL ASENSIO, Roberto. 1994. «La explicitación de la información en la traducción intercultural». En A. Hurtado Albir (ed.) Estudis sobre la traducció. Castellón: UJl: 73-96.

NGUGI WA THIONG'O. 1986. Decolonising the mind. The Politics of Language in African Literature. Portsmouth: Heinemann Education.

NIRANJANA, Tejaswini. 1992. Sitting Translation: History, Post-Structuralism and the Colonial Context. Los Angeles: University of California Press.

NORD, Christiane. 1997. Translating as a Purposeful Activity, Functionalist Approaches Explained. Amsterdam: Rodopi.

PASCUA FEBLES, Isabel. 2017. «El Womanism, visión de la mujer africana en la literatura poscolonial». En Subjetividad, Corporalidad y Necropolítica en la Era Post (posthumanidad, postidentidad, postgénero, postfeminismo...), ed. por Ángeles Mateo. Mato Grosso Do Sul: Revell. Revista de Estudios Literarios UEMS 3 (17): 284-302.

PASCUA FEBLES, Isabel. 2018. «Language and Cultural Identity in Postcolonial African Literature: The Case of Translating Buchi Emecheta into Spanish». En IAFOR. Journal of Arts \& Humanities. 5(2): 69-79. Disponible en https://iafor.org/journal/iafor-journal-of-arts-andhumanities/volume-5-issue-2/article-5/

RodRíGUEZ-GARcíA, Alba. 2015. Para una traducción de la literatura poscolonial africana eurófona: análisis contrastivo francés-español de la escritura femenina de Ken Bugul. Tesis doctoral. Universidad Las Palmas de Gran Canaria. Consultable en http://hdl.handle. net/10553/21688

RODRíGUEZ-GARCíA, Alba. 2018. «Hacia una clasificación del error lingüístico en las traducciones de la literatura africana francófona». En Clases y categorías lingüísticas en contraste, ed. por Elia Hernández Socas, José Juan Batista Rodríguez y Carsten Sinner. Frankfurt am Main: Lang, 157-176.

RODRíGUEZ-GARCíA, Alba. 2019. «Alienating, Naturalizing or Glocalizing?: Toward Blended Translation Strategies for Europhone African Writing» (en prensa).

Sougou, Omar. 2002. Writing Across Cultures: Gender Politics and Difference in the Fiction of Buchi Emecheta. Amsterdam: Rodopi.

Isabel PASCUA FEBLES y Alba RODRÍGUEZ-GARCIAA Traducir las identidades africanas: una mirada mujerista
CLINA

vol. 5-1, June 2019, 95-112

elSSN: 2444-1961

Ediciones Universidad de Salamanca - CC BY-NC-ND 
TYMOCZKO, Maria. 2006. «Translation, Ethics, Ideology, Action». En The Massachusetts Review 47 (3), 442-461.

VENUTI, Lawrence. 1995. The Translator's Invisibility: A History of Translation. London: Routledge. VLÁJOV, SERGUÉl y SIDER FLORIN. 1969. «Neperovodimoe V perevode. Realii». En Masterstvoperevoda 6, 432-456.

VIDAL CLARAMONTE, M. ${ }^{a}$ Carmen África. 2007. Traducir entre culturas. Frankfurt: Peter Lang.

VIDAL CLARAMONTE, M. ${ }^{a}$ Carmen África. 2018. La traducción y la(s) historia(s). Nuevas vías para la investigación. Granada: Editorial Comares.

WITTE, Heidrun. 2017. Blickwechsel. Interkulturelle Wahrnehmung im translatorischen Handeln. Berlin: Frank \& Timme. 